\title{
A NOTE ON NORMALIZATIONS OF ORBIT CLOSURES
}

\author{
JESKO HÜTTENHAIN
}

\begin{abstract}
We give a negative answer to a question by J.M. Landsberg on the nature of normalizations of orbit closures. A counterexample originates from the study of complex, ternary, cubic forms.
\end{abstract}

\section{INTRODUCTION}

We work over the field of complex numbers and $W$ will always denote a finitedimensional $\mathbb{C}$-vector space. Mulmuley and Sohoni [MS01] propose, in their geometric complexity theory, to study the geometry of the orbit closure of certain homogeneous polynomials $P \in \mathbb{C}[W]$ under the action of $\mathrm{GL}(W)$ by precomposition. Unfortunately, the orbit closures are not normal in the main cases of interest [Kum13, BI11]. When $P$ is a product of linear forms, the normalization of the orbit closure has a representation-theoretic description. This observation motivated J.M. Landsberg in [Lan15, Problem 7.19] to pose the following question:

Question 1. "Is it true that whenever a $\mathrm{GL}(W)$-orbit closure with reductive stabilizer has an irreducible boundary, the coordinate ring of the normalization of the orbit closure equals the polynomial part of the coordinate ring of the orbit?"

The remaining undefined terminology will be explained in Section 2. We show that the orbit of an elliptic curve is an example that answers this question in the negative:

Theorem 2. Let $W=\mathbb{C}^{3}$ and let $P \in \mathbb{C}[W]_{3}$ be any form that defines a nonsingular curve in $\mathbb{P}^{2}$. The group $\mathrm{GL}(W)$ acts on $\mathbb{C}[W]_{3}$ by precomposition. Let $\Omega$ be the orbit of $P$ under this action, $\bar{\Omega}$ its Zariski closure and $\nu: \mathrm{N}(\bar{\Omega}) \rightarrow \bar{\Omega}$ the normalization of $\bar{\Omega}$.

Then, the stabilizer of $P$ is reductive, the boundary $\partial \Omega:=\bar{\Omega} \backslash \Omega$ is irreducible and $\mathbb{C}[\mathrm{N}(\bar{\Omega})]$ is not isomorphic to the polynomial part of $\mathbb{C}[\Omega]$.

In Section 1, we quote classical results about ternary cubics to deduce that the stabilizer of $P$ is reductive and the boundary of its orbit irreducible. The main point of this note is to verify the other claims of Theorem 2, which we will do at the end of Section 2. The third section is dedicated to the proof of an auxiliary result that sheds some further light on the context of Question 1. Section 4 illustrates Theorem 2 by example of the Fermat cubic $P=x^{3}+y^{3}+z^{3}$ and its orbit closure, the Aronhold hypersurface.

Acknowledgement. I thank Peter Bürgisser very much for his many helpful comments about this note. I also extend my gratitude to J.M. Landsberg for his encouragement and several related discussions. Finally, I am grateful to Christian Ikenmeyer for providing Formula (5) in Section 4.

2000 Mathematics Subject Classification. 14L24, 13A50.

Key words and phrases. Geometric complexity theory, birational geometry, classical groups. Partially funded by grant BU 1371/2-2 of the Deutsche Forschungsgemeinschaft. 


\section{Elliptic Curves have Irreducible Boundary}

We let $W=\mathbb{C}^{3}$ and consider the space $\mathbb{C}[W]_{3}$ of ternary cubics. GL $(W)$ acts on this space from the right via $\mathbb{C}[W]_{3} \times \mathrm{GL}(W) \rightarrow \mathbb{C}[W]_{3},(Q, g) \mapsto Q \circ g$. For $Q \in \mathbb{C}[W]_{3}$, we denote by $\Omega_{Q}:=Q \circ \mathrm{GL}(W)$ the orbit of $Q$. We define $\bar{\Omega}_{Q}:=\overline{\Omega_{Q}}$ to be its Zariski closure and set $\partial \Omega_{Q}:=\bar{\Omega}_{Q} \backslash \Omega_{Q}$. We say that $P \in \mathbb{C}[W]_{3}$ is regular if its (projective) vanishing set is a smooth plane projective curve. This is equivalent to the condition that for every nonzero $w \in W$, there is a partial derivative of $P$ which does not vanish at $w$. The following is classical and well-known:

Proposition 3. If $P \in \mathbb{C}[W]_{3}$ is regular, it has a finite (hence reductive) stabilizer and the boundary $\partial \Omega_{P}$ is irreducible.

Proof. By Corollary 1 of [KMM02], the stabilizer of $P$ is finite. Any finite group is reductive by Maschke's Theorem. A complete diagram of the degeneracy behaviour of all ternary cubic forms can be found in Section 4 of [KMM02]. Choosing coordinates $\mathbb{C}[W]=\mathbb{C}[x, y, z]$, the diagram implies that $\partial \Omega_{P}$ is equal to the orbit closure of the polynomial $Q:=x^{3}-y^{2} z$, regardless of the choice of $P$. Hence, $\partial \Omega_{P}=\bar{\Omega}_{Q}$ is irreducible: It is the closure of the image of the irreducible variety $\mathrm{GL}(W)$ under the regular map $\mathrm{GL}(W) \rightarrow \mathbb{C}[W]_{3}, g \mapsto Q \circ g$.

\section{The Polynomial Part of a Module}

In analogy to the case of homogeneous polynomials, we consider right actions of $\mathrm{GL}(W)$ on an arbitrary, finite-dimensional $\mathbb{C}$-vector space $\mathbb{V}$. This action will also be denoted by a "○". To explain the notion polynomial part, we require a brief recollection of the representation theory of $\mathrm{GL}(W)$, see [Hum75, Kra84] for some textbooks on the subject. The irreducible representations of $\mathrm{GL}(W) \cong \mathrm{GL}_{n}(\mathbb{C})$ are classified by the semigroup

$$
\Lambda:=\left\{\lambda \in \mathbb{Z}^{n} \mid \lambda_{1} \geq \cdots \geq \lambda_{n}\right\}
$$

of dominant weights. We denote by $\mathbb{V}(\lambda)$ the irreducible $\mathrm{GL}(W)$-module corresponding to the weight $\lambda \in \Lambda$. There is a partial ordering $\sqsubseteq$ on $\Lambda$ defined as follows: We have $\mu \sqsubseteq \lambda$ if and only if $\mu_{i} \leq \lambda_{i}$ for all $1 \leq i \leq n$. The action of $\mathrm{GL}(W)$ on $\mathbb{V}(\lambda)$ extends to a morphism $\mathbb{V}(\lambda) \times \operatorname{End}(W) \rightarrow \mathbb{V}(\lambda)$ of complex varieties if and only if $0 \sqsubseteq \lambda$, where $0 \in \mathbb{Z}^{n}$ denotes the zero vector.

When $\mathbb{V}$ is any GL(W)-module, we can decompose it as $\mathbb{V} \cong \bigoplus_{\lambda \in \Lambda} \mathbb{V}(\lambda)^{\oplus n_{\lambda}}$ for certain $n_{\lambda} \in \mathbb{N}$. We then write

$$
\mathbb{V}_{\sqsupseteq 0}:=\bigoplus_{\substack{\lambda \in \Lambda \\ \lambda \sqsupseteq 0}} \mathbb{V}(\lambda)^{\oplus n_{\lambda}} \subseteq \mathbb{V} .
$$

and call this the polynomial part of $\mathbb{V}$. We say that $\mathbb{V}$ is a polynomial $\mathrm{GL}(W)$ module if $\mathbb{V}=\mathbb{V}_{\sqsupseteq 0}$. We can rephrase Question 1 as follows:

Question. "Let $\mathbb{V}$ be a polynomial $\mathrm{GL}(W)$-module and $P \in \mathbb{V}$. Let $\Omega \subseteq \mathbb{V}$ be the orbit and $H \subseteq \mathrm{GL}(W)$ the stabilizer group of $P$. Assume that $\partial \Omega$ is irreducible and $H$ is reductive. Let $\nu: \mathrm{N}(\bar{\Omega}) \rightarrow \bar{\Omega}$ be the normalization of the orbit closure of $P$. Is there a $\mathrm{GL}(W)$-module isomorphism $\mathbb{C}[\mathrm{N}(\bar{\Omega})] \cong \mathbb{C}[\Omega]]_{0}$ ?"

Theorem 4. Let $\mathbb{V}$ be a polynomial GL(W)-module, $P \in \mathbb{V}$ a point with reductive stabilizer $H \subseteq \mathrm{GL}(W)$. Denote by $\Omega=P \circ \mathrm{GL}(W)$ its orbit and by $\nu: \mathrm{N}(\bar{\Omega}) \rightarrow \bar{\Omega}$ the normalization of its orbit closure. 
There is an injective homomorphism $\iota: \mathbb{C}[\mathrm{N}(\bar{\Omega})] \hookrightarrow \mathbb{C}[\Omega] \sqsupseteq 0$ of graded $\mathbb{C}$-algebras and $\mathrm{GL}(W)$-modules. Futhermore, the following statements are equivalent:

(1) The injection $\iota$ is an isomorphism.

(2) For all $a \in \operatorname{End}(W)$ with $P \circ a=0$, we have $0 \in \overline{H a}$.

If either condition is satisfied, we have $P \circ \operatorname{End}(W)=\bar{\Omega}$.

Section 3 is dedicated to the proof of this statement. We demonstrate how our main result follows from it:

Proof of Theorem 2. By Proposition 3, the polynomial $P$ has a reductive stabilizer and its orbit has an irreducible boundary. Let $[w] \in \mathbb{P}^{2}$ be any point on the curve defined by $P$, i.e. $w \in W$ is nonzero and $P(w)=0$. Let $a \in \operatorname{End}(W)$ be of rank one such that $\operatorname{im}(a)$ is spanned by $w$. Then, $P \circ a=0$ and since $H$ is a finite group, $\overline{H a}=H a$ does not contain the zero map. Hence by Theorem 4 , the two $\operatorname{GL}(W)$-modules $\mathbb{C}[\mathrm{N}(\bar{\Omega})]$ and $\mathbb{C}[\Omega] \sqsupseteq 0$ are not isomorphic.

\section{Proof of Theorem 4}

We recall the algebraic Peter-Weyl Theorem, see [TY05, 27.3.9]:

$$
\mathbb{C}[\mathrm{GL}(W)] \cong \bigoplus_{\lambda \in \Lambda} \mathbb{V}(\lambda) \otimes \mathbb{V}(\lambda)^{*}
$$

Based on this, we make the following fundamental observation:

Lemma 5. The inclusion $\mathrm{GL}(W) \subseteq \operatorname{End}(W)$ is an open, $\operatorname{GL}(W)$-equivariant immersion of varieties under the operation of $\mathrm{GL}(W)$ acting by multiplication from the left on both affine varieties. It induces an inclusion of their respective coordinate rings which satisfies $\mathbb{C}[\operatorname{End}(W)]=\mathbb{C}[\mathrm{GL}(W)]_{\sqsupseteq 0}$.

Proof. By [Lan12, Formula (6.5.1)], we get the last equality in

$$
\mathbb{C}[\operatorname{End}(W)]_{d} \cong \mathbb{C}\left[W \otimes W^{*}\right]_{d} \cong \operatorname{Sym}^{d}\left(W \otimes W^{*}\right) \cong \bigoplus_{\substack{0 \sqsubseteq \lambda \in \Lambda \\ \lambda_{1}+\cdots+\lambda_{n}=d}} \mathbb{V}(\lambda) \otimes \mathbb{V}(\lambda)^{*}
$$

By summing over $d$ and applying (1), we obtain

$$
\mathbb{C}[\operatorname{End}(W)] \cong \bigoplus_{0 \sqsubseteq \lambda \in \Lambda} \mathbb{V}(\lambda) \otimes \mathbb{V}(\lambda)^{*}=\mathbb{C}[\mathrm{GL}(W)]_{\sqsupseteq 0}
$$

by the definition of the polynomial part.

Note that the stabilizer $H$ acts on the variety $\operatorname{End}(W)$ by multiplication from the left. Since $\mathbb{V}$ is a polynomial GL $(W)$-module, there is a well-defined morphism $\omega: \operatorname{End}(W) \rightarrow \bar{\Omega}, a \mapsto P \circ a$. For $h \in H$ and $a \in \operatorname{End}(W)$,

$$
\omega(h a)=P \circ(h a)=P \circ h \circ a=P \circ a=\omega(a) .
$$

Therefore, $\omega$ is an $H$-invariant morphism. Refer to [TY05, 27.5.1] for the following fact: Since $H$ is a reductive algebraic group, there is an affine quotient variety $\operatorname{End}(W) / / H$ together with a surjective morphism $\pi: \operatorname{End}(W) \rightarrow \operatorname{End}(W) / / H$ and $\omega$ factors as a morphism $\phi: \operatorname{End}(W) / / H \rightarrow \bar{\Omega}$ such that the following diagram commutes:

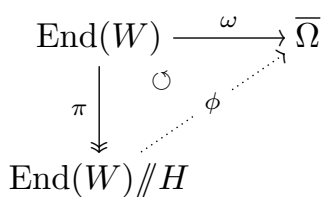


Furthermore, the variety $\operatorname{End}(W) / / H$ is a normal variety because $\operatorname{End}(W)$ is normal [TY05, 27.5.1].

The morphism $\phi$ is a birational map. Indeed, $\operatorname{End}(W) / / H$ contains $\operatorname{GL}(W) / / H$ as an open subset [TY05, 27.5.2] and the restriction of $\phi$ to this open subset is the isomorphism $\mathrm{GL}(W) / / H \cong \Omega$, [TY05, 25.4.6]. Since $\Omega$ is an open subset of its closure [TY05, 21.4.3], this proves that $\phi$ is generically one to one.

The normalization $\nu: \mathrm{N}(\bar{\Omega}) \rightarrow \bar{\Omega}$ is a surjective, finite morphism of affine algebraic varieties [GW10, Proposition 12.43 and Corollary 12.52]. By the universal property of the normalization [GW10, Corollary 12.45] there exists a unique morphism $\psi: \operatorname{End}(W) / / H \rightarrow \mathrm{N}(\bar{\Omega})$ which completes (2) to a commutative diagram:

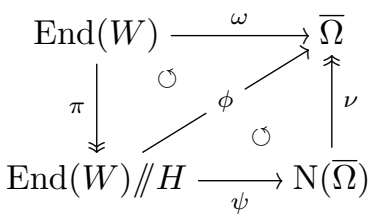

The morphism $\psi$ is dominant and therefore corresponds to an injective ring homomorphism

$$
\mathbb{C}[\mathrm{N}(\bar{\Omega})] \subseteq \mathbb{C}[\operatorname{End}(W) / / H]=\mathbb{C}[\operatorname{End}(W)]^{H}=\left(\mathbb{C}[\mathrm{GL}(W)]_{\sqsupseteq 0}\right)^{H},
$$

due to Lemma 5. Taking $H$-invariants is with respect to the left action of $H$ on $\mathbb{C}[\mathrm{GL}(W)]$ and considering polynomial submodules is with respect to the right action of $\mathrm{GL}(W)$ on $\mathbb{C}[\mathrm{GL}(W)]$, so these two operations commute. Hence,

$$
\mathbb{C}[\mathrm{N}(\bar{\Omega})] \subseteq\left(\mathbb{C}[\mathrm{GL}(W)]^{H}\right)_{\sqsupseteq 0}=\mathbb{C}[\Omega]_{\sqsupseteq 0} .
$$

In other words, the polynomial part of the coordinate ring of the orbit of $P$ is the ring of $H$-invariants in $\mathbb{C}[\operatorname{End}(W)]$, where $H$ is the stabilizer of $P$.

Summary. There is a commutative diagram of $\mathrm{GL}(W)$-equivariant inclusions of C-algebras:

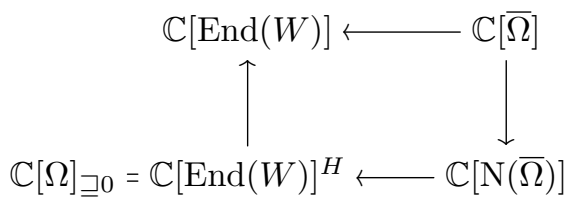

Here, $\mathbb{C}[\bar{\Omega}]$ and $\mathbb{C}[\operatorname{End}(W)]^{H}$ have the same quotient field $\mathbb{K}$. The inclusion

$$
\mathbb{C}[\mathrm{N}(\bar{\Omega})] \subseteq \mathbb{C}[\operatorname{End}(W)]^{H}=\mathbb{C}[\Omega]_{\sqsupseteq 0}
$$

is an inclusion of integrally closed subrings of $\mathbb{K}$. (By definition, $\mathbb{C}[\mathrm{N}(\bar{\Omega})]$ is the integral closure of $\mathbb{C}[\bar{\Omega}]$ in $\mathbb{K}$.)

We now show that (1) of Theorem 4 implies $P \circ \operatorname{End}(W)=\bar{\Omega}$. Recall (3). The condition $\mathbb{C}[\mathrm{N}(\bar{\Omega})]=\mathbb{C}[\Omega]_{\sqsupseteq 0}$ holds if and only if the morphism $\psi$ is an isomorphism. In this case it follows that $\phi$ is the normalization of $\bar{\Omega}$. Thus, (1) implies in particular that $\phi$ is surjective and therefore $\omega$ is surjective, which means $P \circ \operatorname{End}(W)=\bar{\Omega}$.

We now ask when the inclusion $\mathbb{C}[\mathrm{N}(\bar{\Omega})] \subseteq \mathbb{C}[\Omega]_{\sqsupseteq 0}$ becomes an equality. We will require an auxiliary lemma for the proof. Recall that the algebraic group $\mathbb{C}^{\times}=\mathrm{GL}_{1}$ acts polynomially on a variety $X$ if the action morphism $\mathbb{C}^{\times} \times X \rightarrow X$ lifts to a morphism $\mathbb{C} \times X \rightarrow X$. We will denote this map by a dot, i.e. $(t, x) \mapsto t . x$.

Lemma 6. Let $X$ and $Y$ be affine varieties, each of them equipped with polynomial $\mathbb{C}^{\times}$-actions admitting unique fixed points $0_{X} \in X$ and $0_{Y} \in Y$, respectively. Let 
$\phi: X \rightarrow Y$ be a $\mathbb{C}^{\times}$-equivariant morphism. Then, $\phi^{-1}\left(0_{Y}\right)=\left\{0_{X}\right\}$ if and only if $\phi$ is finite.

Proof. The "only if" part is [Lan15, Lemma 7.6.3]. For the converse, assume that $\phi$ is finite. Let $x \in X$ be such that $\phi(x)=0_{Y}$. Then, $\phi(t . x)=t . \phi(x)=t \cdot 0_{Y}=0_{Y}$ for all $t \in \mathbb{C}^{\times}$and hence, $\mathbb{C}^{\times} . x \subseteq \phi^{-1}\left(0_{Y}\right)$. But $\phi^{-1}\left(0_{Y}\right)$ is a finite set, therefore $\mathbb{C}^{\times} \cdot x$ is finite and irreducible, i.e. a point. This implies $\mathbb{C}^{\times} \cdot x=\{x\}$, so $x$ is a fixpoint for the action of $\mathbb{C}^{\times}$. It follows that $x=0_{X}$ by uniqueness of the fixpoint.

Lemma 6 will be applied to the morphism $\phi: \operatorname{End}(W) / / H \rightarrow \bar{\Omega}$. We therefore study the action of the scalar matrices $\mathbb{C}^{\times} \subseteq \mathrm{GL}(W)$ on $\operatorname{End}(W) / / H$ and $\bar{\Omega}$. Observe that the morphism $\phi$ is equivariant with respect to this action. We need to make sure that both varieties have a unique fixpoint in order to make use of Lemma 6.

We first reduce to the case where $\mathbb{V}$ has a unique fixpoint under the action of all scalar matrices. For this purpose, fix some basis of $W$, so $\mathrm{GL}(W) \cong \mathrm{GL}_{n}(\mathbb{C})$ and let $\mathbb{V}=\bigoplus_{\lambda \in \mathbb{N}^{n}} \mathbb{V}_{\lambda}$ be the decomposition of $\mathbb{V}$ into isotypical components, i.e. $\mathbb{V}_{\lambda}$ is a direct sum of irreducible modules of type $\lambda$. Note that the only weights $\lambda$ that appear are in $\mathbb{N}^{n}$ because $\mathbb{V}$ is a polynomial $\mathrm{GL}_{n}(\mathbb{C})$-module. Let $P=\sum_{\lambda \in \mathbb{N}^{n}} P_{\lambda}$ be the corresponding decomposition of $P$, i.e. $P_{\lambda} \in \mathbb{V}_{\lambda}$. Observe that the point $\tilde{P}:=P-P_{0}$ has the same stabilizer as $P$, because any element of $\mathbb{V}_{0}$ is $\operatorname{GL}(W)$-invariant. Let $\tilde{\mathbb{V}}:=\bigoplus_{\lambda \neq 0} \mathbb{V}_{\lambda}$ be the complement of $\mathbb{V}_{0}$ in $\mathbb{V}$. Then, $\Omega=\left\{P_{0}\right\} \times \Omega_{\tilde{P}} \subseteq \mathbb{V}_{0} \times \tilde{\mathbb{V}}=\mathbb{V}$ and consequently, $\bar{\Omega}_{P}=\left\{P_{0}\right\} \times \bar{\Omega}_{\tilde{P}} \cong \bar{\Omega}_{\tilde{P}}$. This shows that we may henceforth assume $\mathbb{V}=\tilde{\mathbb{V}}$ and $P=\tilde{P}$. In this situation, the origin $0_{\mathbb{V}} \in \mathbb{V}$ is the only fixpoint under the action of the scalar matrices. Consequently, it is also the only $\mathbb{C}^{\times}$-fixpoint in $\bar{\Omega}$.

On the other hand, $\operatorname{End}(W)$ also has a unique fixpoint with respect to the left action by scalar matrices, namely the zero map which we will denote by 0 . At this point, we require the following lemma to deduce that $\operatorname{End}(W) / / H$ also has a unique fixpoint:

Lemma 7. Let $E$ be an affine variety on which $\mathbb{C}^{\times}$acts polynomially with a unique fixpoint 0. Assume that a reductive group $H$ acts on $E$ from the left such that the actions of $H$ and $\mathbb{C}^{\times}$commute. Then, the quotient $E / / H$ also has a unique fixpoint under the induced action of $\mathbb{C}^{\times}$.

The proof of this lemma is slightly technical and will be given afterwards. Using it, we conclude that $\pi(0)$ is the unique fixpoint in $X:=\operatorname{End}(W) / / H$ and $0_{\mathbb{V}}$ is the unique fixpoint in $Y:=\bar{\Omega}$. The morphism $\phi: X \rightarrow Y$ now satisfies the conditions of Lemma 6.

We proceed to prove the equivalence of (1) and (2).

We now show $(1) \Rightarrow(2)$. If $(1)$ holds, $\psi$ is an isomorphism and $\phi$ is a normalization of $\bar{\Omega}$. Therefore, $\phi$ is a finite morphism. By one direction of Lemma 6 , this implies that $\phi^{-1}\left(0_{\mathbb{V}}\right)=\{\pi(0)\}$. In other words, $\phi(\pi(a))=0_{\mathbb{V}}$ implies $\pi(a)=\pi(0)$. We have $P \circ a=\omega(a)=\phi(\pi(a))$, so $P \circ a=0_{\mathbb{V}}$ implies $\pi(a)=\pi(0)$, which is the same as saying that the zero map 0 is contained in the closure of the $H$-orbit of $a$. This is precisely (2).

For the converse implication, we assume (2). For any $a \in \operatorname{End}(W)$, the condition $0_{\mathbb{V}}=\phi(\pi(a))=\omega(a)$ implies $0 \in \overline{H a}$ by (2). By construction of the GIT quotient, this implies $\pi(a)=\pi(0)$ and hence, $\phi^{-1}\left(0_{\mathbb{V}}\right)=\{\pi(0)\}$. The other direction of Lemma 6 now states that $\phi$ is a finite morphism. Any finite morphism is 
integral [GW10, Remark 12.10], so $\phi$ is an integral birational map from a normal variety $\operatorname{End}(W) / / H$ to $\bar{\Omega}$. By [GW10, Proposition 12.44], it follows that it is the normalization of $\bar{\Omega}$, so $\psi$ is an isomorphism.

Proof of Lemma \%. We first note that 0 is a fixpoint for the action of $H$ as well. Indeed, for any $h \in H$ and any $t \in \mathbb{C}^{\times}$, we have t.h.0 =h.t.0 $=$h.0 because the actions commute, so $h .0$ is a fixpoint for the action of $\mathbb{C}^{\times}$. By uniqueness, this implies $h .0=0$. As $h \in H$ was arbitrary, 0 is a fixpoint for the action of $H$.

We will denote by $\pi: E \rightarrow E / / H$ the quotient morphism. Assume now that $x:=\pi(e) \in E / / H$ is any fixpoint of the action of $\mathbb{C}^{\times}$. Observe that

$$
\{x\}=\mathbb{C}^{\times} \cdot x=\mathbb{C}^{\times} \cdot \pi(e)=\pi\left(\mathbb{C}^{\times} \cdot e\right),
$$

so $\mathbb{C}^{\times} . e \subseteq \pi^{-1}(x)$. Since the action of $\mathbb{C}^{\times}$is polynomial, the orbit map lifts to a $\mathbb{C}^{\times}$-equivariant morphism $\gamma: \mathbb{C} \rightarrow E$ with $\gamma(t)=$ t.e for $t \in \mathbb{C}^{\times}$. Since

$$
t . \gamma(0)=\gamma(t \cdot 0)=\gamma(0)
$$

for all $t \in \mathbb{C}^{\times}$, it follows that $\gamma(0)$ is a $\mathbb{C}^{\times}$-fixpoint in $E$, so $\gamma(0)=0$. This implies that $0 \in \overline{\mathbb{C}^{\times}} . e$. Because 0 is a fixpoint for the action of $H$, the set $\{0\} \subseteq E$ is a closed $H$-orbit. By the nature of the GIT-quotient, points of $E$ that share a closed $H$-orbit are mapped to the same point in the quotient. In this case, $e$ and 0 share the closed orbit $\{0\}$ and it follows that $x=\pi(e)=\pi(0)$. Thus, $\pi(0)$ is the only fixpoint of the action of $\mathbb{C}^{\times}$on $E / / H$.

\section{Example: The Aronhold Hypersurface}

As an example we consider the special case of the Fermat cubic: Let $W=\mathbb{C}^{3}$, $\mathbb{V}=\mathbb{C}[W]_{3}=\mathbb{C}[x, y, z]_{3}$ and $P:=x^{3}+y^{3}+z^{3} \in \mathbb{V}$. By Theorem 2 and 4 , we know that the quotient $\mathbb{C}[\Omega]_{\sqsupseteq 0} / \mathbb{C}[\mathrm{N}(\bar{\Omega})]$ exists and that it is a nontrivial $\mathrm{GL}_{3}$-module, so it decomposes as a direct sum of irreducible $\mathrm{GL}_{3}$-modules. We will explicitly compute some of the corresponding multiplicities.

Note that this is a special case as the orbit closure $\bar{\Omega} \subseteq \mathbb{V}$ is a normal variety [IK99, Thm. 1.56]. This simplifies the calculation because we need not determine the normalization of $\bar{\Omega}$. Note that if $P$ is a generic regular cubic, $\bar{\Omega}$ is not normal [BI15, Cor. 3.17 (1)].

$P$ defines the elliptic curve with $j$-invariant equal to zero [Stu93, Prop. 4.4.7 and eq. (4.5.8)]. Its orbit closure $\bar{\Omega}$ is the hypersurface defined by the Aronhold Invariant $\mathcal{A} \in \mathbb{C}[\mathbb{V}]_{4}$, see [DK93, 5.31.1] and [IK99, Remark 7.20]. Thus,

$$
\mathbb{C}[\mathrm{N}(\bar{\Omega})]=\mathbb{C}[\bar{\Omega}]=\mathbb{C}[\mathbb{V}] /\langle\mathcal{A}\rangle .
$$

We write $\operatorname{Sym}^{d} \operatorname{Sym}^{m} \mathbb{C}^{3}=\bigoplus_{\lambda \in \Lambda} \mathbb{V}(\lambda)^{\mathrm{p}(d, m ; \lambda)}$, where $\mathrm{p}(d, m ; \lambda)$ is known as a Plethysm coefficient. Furthermore, $\mathcal{A}$ is a highest weight vector of weight $(4,4,4)$ with respect to the action of $\mathrm{GL}(W) \cong \mathrm{GL}_{3}(\mathbb{C})$. This means that the linear span of the $\mathrm{GL}_{3}$-orbit of $\mathcal{A}$ is isomorphic to the irreducible $\mathrm{GL}_{3}$-module $\mathbb{V}((4,4,4))$. Thus, $\langle\mathcal{A}\rangle_{d}=\mathbb{C}[\mathbb{V}]_{d-4} \cdot \mathcal{A}=\bigoplus_{\lambda \in \Lambda} \mathbb{V}(\lambda)^{\mathrm{p}(d-4,3 ; \lambda-(4,4,4))}$ and

$$
\mathbb{C}[\mathrm{N}(\bar{\Omega})]_{d} \cong \bigoplus_{\lambda \in \Lambda} \mathbb{V}(\lambda)^{b_{\lambda}}
$$

where $b_{\lambda}=\mathrm{p}(d, 3 ; \lambda)-\mathrm{p}(d-4,3 ; \lambda-(4,4,4))$. Note that $b_{\lambda}$ can be computed with the SCHUR software package (http://schur.sourceforge.net/). 


\begin{tabular}{|c|c|c|c|c|c|c|c|c|c|c|c|c|}
\hline$a_{\lambda}$ & & $\lambda$ & & $b_{\lambda}$ & $n_{\lambda} \lambda$ & & $b_{\lambda}$ & $m_{\lambda}$ & & & $\lambda$ & $n_{\lambda} \lambda$ \\
\hline 1 & 1 & $0(4,2,0)$ & 1 & 1 & $0(8,8,2)$ & 4 & 3 & & $(12,6,3)$ & 2 & 2 & $0(13,7,4)$ \\
\hline 2 & 1 & $1(6,0,0)$ & 2 & 2 & $0(9,6,3)$ & 4 & 2 & 2 & $(12,7,2)$ & 4 & 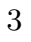 & $1(13,8,3)$ \\
\hline 1 & 1 & $0(4,4,1)$ & 1 & 1 & $0(9,7,2)$ & 5 & 1 & & $(12,8,1)$ & 5 & 2 & $3(13,9,2)$ \\
\hline 1 & 1 & $0(5,2,2)$ & 1 & 0 & $1(9,8,1)$ & 6 & 1 & & $(12,9,0)$ & 5 & 1 & $4(13,10,1)$ \\
\hline 2 & 1 & $1(6,3,0)$ & 1 & 0 & $1(9,9,0)$ & 1 & 1 & & $(13,4,4)$ & 4 & 0 & $4(13,11,0)$ \\
\hline 2 & 1 & $1(7,2,0)$ & 1 & 1 & $0(10,4,4)$ & 2 & 1 & & $(13,5,3)$ & 4 & 3 & $1(14,6,4)$ \\
\hline 1 & 0 & $1(8,1,0)$ & 1 & 1 & $0(10,5,3)$ & 6 & 3 & 3 & $(13,6,2)$ & 4 & 2 & $2(14,7,3)$ \\
\hline 3 & 1 & $2(9,0,0)$ & 3 & 2 & $1(10,6,2)$ & 5 & 1 & & $(13,7,1)$ & 7 & 3 & $4(14,8,2)$ \\
\hline 1 & 1 & $0(6,4,2)$ & 3 & 1 & $2(10,7,1)$ & 8 & 1 & & $(13,8,0)$ & 7 & 1 & $6(14,9,1)$ \\
\hline 2 & 1 & $1(6,6,0)$ & 4 & 1 & $3(10,8,0)$ & 3 & 1 & & $(14,4,3)$ & 9 & 1 & $8(14,10,0)$ \\
\hline 1 & 1 & $0(7,3,2)$ & 1 & 1 & $0(11,4,3)$ & 5 & 2 & 3 & $(14,5,2)$ & 2 & 1 & $1(15,5,4)$ \\
\hline 1 & 1 & $0(7,4,1)$ & 3 & 2 & $1(11,5,2)$ & 8 & 1 & & $(14,6,1)$ & 6 & 3 & $3(15,6,3)$ \\
\hline 1 & 0 & $1(7,5,0)$ & 4 & 1 & $3(11,6,1)$ & 9 & 1 & & $(14,7,0)$ & 7 & 3 & $4(15,7,2)$ \\
\hline 1 & 1 & $0(8,2,2)$ & 3 & 0 & $3(11,7,0)$ & 1 & 0 & & $(15,3,3)$ & 9 & 1 & $8(15,8,1)$ \\
\hline 1 & 0 & $1(8,3,1)$ & 1 & 0 & $1(12,3,3)$ & 6 & 2 & & $(15,4,2)$ & 11 & 1 & $10(15,9,0)$ \\
\hline 3 & 1 & $2(8,4,0)$ & 4 & 2 & $2(12,4,2)$ & 6 & 0 & 6 & $(15,5,1)$ & 2 & 1 & $1(16,4,4)$ \\
\hline 1 & 0 & $1(9,2,1)$ & 3 & 0 & $3(12,5,1)$ & 13 & 2 & 11 & $(15,6,0)$ & 4 & 1 & $3(16,5,3)$ \\
\hline 3 & 1 & $2(9,3,0)$ & 9 & 2 & $7(12,6,0)$ & 5 & 1 & & $(16,3,2)$ & 10 & 3 & $7(16,6,2)$ \\
\hline 4 & 1 & $3(10,2,0)$ & 3 & 1 & $2(13,3,2)$ & 8 & 1 & 7 & $(16,4,1)$ & 10 & 1 & $9(16,7,1)$ \\
\hline 2 & 0 & $2(11,1,0)$ & 5 & 1 & $4(13,4,1)$ & 12 & 1 & 11 & $(16,5,0)$ & 15 & 2 & $13(16,8,0)$ \\
\hline 4 & 1 & $3(12,0,0)$ & 7 & 1 & $6(13,5,0)$ & 4 & 1 & & $(17,2,2)$ & 4 & 1 & $3(17,4,3)$ \\
\hline 1 & 1 & $0(6,6,3)$ & 3 & 1 & $2(14,2,2)$ & 6 & 0 & 6 & $(17,3,1)$ & 8 & 2 & $6(17,5,2)$ \\
\hline 1 & 1 & $0(7,6,2)$ & 4 & 0 & $4(14,3,1)$ & 13 & 1 & 12 & $(17,4,0)$ & 12 & 1 & $11(17,6,1)$ \\
\hline 1 & 1 & $0(8,4,3)$ & 9 & 1 & $8(14,4,0)$ & 5 & 0 & 5 & $(18,2,1)$ & 14 & 1 & $13(17,7,0)$ \\
\hline 1 & 1 & $0(8,5,2)$ & 3 & 0 & $3(15,2,1)$ & 13 & 1 & 12 & $(18,3,0)$ & 2 & 0 & $2(18,3,3)$ \\
\hline 2 & 1 & $1(8,6,1)$ & 9 & 1 & $8(15,3,0)$ & 1 & 0 & & $(19,1,1)$ & 9 & 2 & $7(18,4,2)$ \\
\hline 1 & 0 & $1(8,7,0)$ & 1 & 0 & $1(16,1,1)$ & 12 & 1 & 11 & $(19,2,0)$ & 10 & 0 & $10(18,5,1)$ \\
\hline 2 & 2 & $0(9,4,2)$ & 9 & 1 & $8(16,2,0)$ & 8 & 0 & 8 & $(20,1,0)$ & 20 & 2 & $18(18,6,0)$ \\
\hline 1 & 0 & $1(9,5,1)$ & 5 & 0 & $5(17,1,0)$ & 8 & 1 & & $(21,0,0)$ & 7 & 1 & $6(19,3,2)$ \\
\hline 4 & 1 & $3(9,6,0)$ & 7 & 1 & $6(18,0,0)$ & 1 & 1 & & $(10,8,6)$ & 11 & 1 & $10(19,4,1)$ \\
\hline 2 & 1 & $1(10,3,2)$ & 1 & 1 & $0(9,6,6)$ & 1 & 1 & & $(10,9,5)$ & 17 & 1 & $16(19,5,0)$ \\
\hline 3 & 1 & $2(10,4,1)$ & 1 & 1 & $0(9,8,4)$ & 1 & 1 & & $(10,10,4)$ & 5 & 1 & $4(20,2,2)$ \\
\hline 4 & 1 & $3(10,5,0)$ & 1 & 1 & $0(10,6,5)$ & 1 & 1 & & $(11,8,5)$ & 9 & 0 & $9(20,3,1)$ \\
\hline 2 & 1 & $1(11,2,2)$ & 1 & 1 & $0(10,7,4)$ & 1 & 1 & & $(11,9,4)$ & 19 & 1 & $18(20,4,0)$ \\
\hline 2 & 0 & $2(11,3,1)$ & 2 & 2 & $0(10,8,3)$ & 1 & 1 & & $(11,10,3)$ & 7 & 0 & $7(21,2,1)$ \\
\hline 5 & 1 & $4(11,4,0)$ & 2 & 1 & $1(10,9,2)$ & 2 & 2 & & $(12,6,6)$ & 17 & 1 & $16(21,3,0)$ \\
\hline 2 & 0 & $2(12,2,1)$ & 2 & 1 & $1(10,10,1)$ & 1 & 1 & & $(12,7,5)$ & 2 & 0 & $2(22,1,1)$ \\
\hline 6 & 1 & $5(12,3,0)$ & 2 & 2 & $0(11,6,4)$ & 3 & 3 & & $(12,8,4)$ & 16 & 1 & $15(22,2,0)$ \\
\hline 6 & 1 & $5(13,2,0)$ & 1 & 1 & $0(11,7,3)$ & 3 & 2 & & $(12,9,3)$ & 10 & 0 & $10(23,1,0)$ \\
\hline 4 & 0 & $4(14,1,0)$ & 3 & 2 & $1(11,8,2)$ & 4 & 2 & & $(12,10,2)$ & 10 & 1 & $9(24,0,0)$ \\
\hline 5 & 1 & $4(15,0,0)$ & 2 & 0 & $2(11,9,1)$ & 2 & 0 & & $(12,11,1)$ & & & \\
\hline 1 & 1 & $0(6,6,6)$ & 2 & 0 & $2(11,10,0)$ & 4 & 1 & & $(12,12,0)$ & & & \\
\hline 1 & 1 & $0(8,6,4)$ & 1 & 1 & $0(12,5,4)$ & 2 & 2 & & $(13,6,5)$ & & & \\
\hline
\end{tabular}

FiguRE 1. Multiplicities in $\mathbb{C}[\Omega]_{\sqsupseteq 0} / \mathbb{C}[N(\bar{\Omega})]$ for the Fermat cubic, up to degree 8 . Column-wise grouped by degree.

Denoting by $a_{\lambda}$ the coefficients such that $\mathbb{C}[\Omega]_{\sqsupseteq 0}=\bigoplus_{\lambda \in \Lambda} \mathbb{V}(\lambda)^{a_{\lambda}}$, we are interested in the numbers $m_{\lambda}:=a_{\lambda}-b_{\lambda}$ because

$$
\mathbb{C}[\Omega]_{\sqsupseteq 0} / \mathbb{C}[\mathrm{N}(\bar{\Omega})]=\bigoplus_{\lambda \in \Lambda} \mathbb{V}(\lambda)^{a_{\lambda}-b_{\lambda}} .
$$

It follows from the Peter-Weyl Theorem (1) that $a_{\lambda}$ is the space of $H$-invariants of $\mathbb{V}(\lambda)$, where $H \subseteq \mathrm{GL}_{3}$ is the stabilizer of $P$. It is well-known [BI15, Prop. 2.4] that $H$ consists of permutation matrices and diagonal matrices whose diagonal entries 
are third roots of unity. One can obtain a matrix representation of the canonical projection $\mathbb{V}(\lambda) \rightarrow \mathbb{V}(\lambda)^{H}$ over the basis of semistandard Young tableaux (SSYT) by symmetrizing each SSYT with respect to $H$ and straightening it [Ful97, § 7.4]. The quantity $a_{\lambda}$ arises as the rank of this matrix. We have computed the values of the $m_{\lambda}:=a_{\lambda}-b_{\lambda}$ up to degree 8 , see Figure 1.

A formula for $a_{\lambda}$ is more involved than the one for $b_{\lambda}$. Advancing methods used in [BI11, Section 4.2] (see also [Ike12, Section 5.2]), Ikenmeyer [Ike16] determined such a formula: For $\lambda \in \Lambda$, denote by $|\lambda|:=\lambda_{1}+\lambda_{2}+\lambda_{3}$ the sum of its entries. We have $a_{\lambda}=0$ unless $|\lambda|=3 d$ for some $d \in \mathbb{N}$. In this case,

$$
a_{\lambda}=\sum_{\substack{\mu \in \Lambda \\|\mu|=d}} \sum_{\substack{\nu_{1}, \ldots, \nu_{d} \in \Lambda,\left|\nu_{k}\right|=3 \cdot k \cdot \hat{\mu}_{k} \\ \text { for all } k}} \mathrm{c}_{\nu_{1}, \ldots, \nu_{d}}^{\lambda} \cdot \prod_{k=1}^{d} \mathrm{p}\left(\hat{\mu}_{k}, 3 k ; \nu_{k}\right),
$$

where $\mathrm{c}_{\nu_{1}, \ldots, \nu_{d}}^{\lambda}$ denotes the multi-Littlewood-Richardson coefficient and $\hat{\mu}_{k}$ denotes the number of times that $k$ appears as an entry of $\mu$.

\section{REFERENCES}

[BI11] Peter Bürgisser and Christian Ikenmeyer. Geometric complexity theory and tensor rank [extended abstract]. In STOC'11-Proceedings of the 43rd ACM Symposium on Theory of Computing, pages 509-518. ACM, New York, 2011.

[BI15] Peter Bürgisser and Christian Ikenmeyer. Fundamental invariants of orbit closures. arXiv 1511.02927, 112015.

[DK93] Igor Dolgachev and Vassil Kanev. Polar covariants of plane cubics and quartics. Adv. Math., 98(2):216-301, 1993.

[Ful97] William Fulton. Young tableaux, volume 35 of London Mathematical Society Student Texts. Cambridge University Press, Cambridge, 1997. With applications to representation theory and geometry.

[GW10] Ulrich Görtz and Torsten Wedhorn. Algebraic geometry I. Advanced Lectures in Mathematics. Vieweg + Teubner, Wiesbaden, 2010. Schemes with examples and exercises.

[Hum75] James E. Humphreys. Linear algebraic groups. Springer-Verlag, New York-Heidelberg, 1975. Graduate Texts in Mathematics, No. 21.

[IK99] Anthony Iarrobino and Vassil Kanev. Power sums, Gorenstein algebras, and determinantal loci, volume 1721 of Lecture Notes in Mathematics. Springer-Verlag, Berlin, 1999. Appendix C by Iarrobino and Steven L. Kleiman.

[Ike12] Christian Ikenmeyer. Geometric Complexity Theory, Tensor Rank, and Littlewood-Richardson Coefficients. Dissertation, Universität Paderborn, 2012.

[Ike16] Christian Ikenmeyer. Private communication, 2016.

[KMM02] Irina A. Kogan and Marc Moreno Maza. Computation of canonical forms for ternary cubics. In Proceedings of the 2002 International Symposium on Symbolic and Algebraic Computation, pages 151-160 (electronic). ACM, New York, 2002.

[Kra84] Hanspeter Kraft. Geometrische Methoden in der Invariantentheorie. Aspects of Mathematics, D1. Friedr. Vieweg \& Sohn, Braunschweig, 1984.

[Kum13] Shrawan Kumar. Geometry of orbits of permanents and determinants. Comment. Math. Helv., 88(3):759-788, 2013.

[Lan12] Joseph M. Landsberg. Tensors: geometry and applications, volume 128 of Graduate Studies in Mathematics. American Mathematical Society, Providence, RI, 2012.

[Lan15] Joseph M. Landsberg. Geometric complexity theory: an introduction for geometers. Ann. Univ. Ferrara, 61(1):65-117, 2015.

[MS01] Ketan D. Mulmuley and Milind Sohoni. Geometric complexity theory. I. An approach to the P vs. NP and related problems. SIAM J. Comput., 31(2):496-526, 2001.

[Stu93] Bernd Sturmfels. Algorithms in invariant theory. Texts and Monographs in Symbolic Computation. Springer-Verlag, Vienna, 1993. 
[TY05] Patrice Tauvel and Rupert W. T. Yu. Lie algebras and algebraic groups. Springer Monographs in Mathematics. Springer-Verlag, Berlin, 2005.

Technische Universität Berlin, Strasse des 17. Juni 136, 10623 Berlin, Germany

E-mail address, J. Hüttenhain: jesko@math.tu-berlin.de 